Ultra-wideband Radar Methods and Techniques of Medical Sensing and Imaging

C. N. Paulson, J. T. Chang, C. E. Romero, J. Watson, F. J. Pearce, N. Levin

October 10, 2005

SPIE International Symposium on Optics East Boston, MA, United States October 23, 2005 through October 26, 2005 
This document was prepared as an account of work sponsored by an agency of the United States Government. Neither the United States Government nor the University of California nor any of their employees, makes any warranty, express or implied, or assumes any legal liability or responsibility for the accuracy, completeness, or usefulness of any information, apparatus, product, or process disclosed, or represents that its use would not infringe privately owned rights. Reference herein to any specific commercial product, process, or service by trade name, trademark, manufacturer, or otherwise, does not necessarily constitute or imply its endorsement, recommendation, or favoring by the United States Government or the University of California. The views and opinions of authors expressed herein do not necessarily state or reflect those of the United States Government or the University of California, and shall not be used for advertising or product endorsement purposes. 


\title{
Ultra-wideband Radar Methods and Techniques of Medical Sensing and Imaging
}

\author{
Christine N. Paulson ${ }^{*}$, John T. Chang ${ }^{\mathrm{a}}$, Carlos E. Romero ${ }^{\mathrm{a}}$, Joe Watson ${ }^{\mathrm{b}}$, FJ Pearce ${ }^{\mathrm{c}}$, Nathan Levin ${ }^{\mathrm{d}}$ \\ ${ }^{a}$ Lawrence Livermore National Laboratory, 7000 East Avenue, Livermore, CA USA 94550 \\ ${ }^{\mathrm{b}}$ UC Davis Medical Center, 2315 Stockton Blvd, Sacramento, CA 95817 \\ ${ }^{c}$ Walter Reed Army Institute of Research, 503 Robert Grant Ave, Silver Spring, MD. 20910 \\ ${ }^{\mathrm{d}}$ Renal Research Institute, 207 East 94th Street, Suite 303, New York, NY 10128
}

\begin{abstract}
Ultra-wideband radar holds great promise for a variety of medical applications. We have demonstrated the feasibility of using ultra-wideband sensors for detection of internal injuries, monitoring of respiratory and cardiac functions, and continuous non-contact imaging of the human body. Sensors are low-power, portable, and do not require physical contact with the patient. They are ideal for use by emergency responders to make rapid diagnosis and triage decisions. In the hospital, vital signs monitoring and imaging application could improve patient outcomes. In this paper we present an overview of ultra-wideband radar technology, discuss key design tradeoffs, and give examples of ongoing research in applying ultra-wideband technology to the medical field.
\end{abstract}

Keywords: Ultra-wideband, UWB, Micro-power impulse radar, medical, hematoma, cardiac, respiratory, imaging

\section{INTRODUCTION}

Motion sensing and imaging ultra-wideband (UWB) radar systems have strong potential for use in the medical field. UWB radar is non-invasive, low power, and can be manufactured in a small, portable form factor. We have demonstrated the use of UWB radar in a variety of medical applications. A handheld UWB sensor has demonstrated the feasibility of detecting the presence of traumatic internal injuries including intracranial hematoma and pneumothorax. A UWB vital signs monitor tracks cardiac and respiratory motions remotely, without direct skin contact. UWB radar arrays can image the human body. This paper will explore key tradeoffs in the design of ultra-wideband systems for medical applications and provide several examples of research in this area.

\subsection{Ultra-wideband Basics}

Unlike narrowband systems, which transmit continuous waveforms at a specific frequency, ultra-wideband systems transmit narrow impulse-like signals that span a broad frequency range [Figure 1]. The pulse width of a UWB system is typically within a range of 100 's of picoseconds to several nanoseconds, with rise times as fast as 50 picoseconds, corresponding to a frequency range that can span several gigahertz. Since the energy of the pulse is distributed across a many frequencies, the power spectral density is much lower in magnitude than a narrowband system. To a narrowband system, ultra-wideband signals appear below the noise floor, and are therefore very difficult to detect.

UWB signals can be used in many applications, including imaging, vehicular radar, and communications. Examples of imaging applications are ground penetrating radar, through-wall imaging to detect the location or movements of objects, surveillance, search and rescue, and medical systems. Vehicular radar systems are commonly used for collision avoidance and roadside assistance. Ultra-wideband communication systems are useful for high data rate transmission in harsh propagation environments, such as indoor applications with dense multi-path channels, for consumer electronics, and for covert operations.

The FCC defines ultra-wideband signals to be those with fractional bandwidth exceeding $20 \%$ of the center frequency, or those with greater than $500 \mathrm{MHz}$ bandwidth ${ }^{1}$. Although development of ultra-wideband technologies began in the

\footnotetext{
*paulson4@1lnl.gov (925) 423-7362 www.llnl.gov $\quad$ UCRL-XXXXXXX
} 
$1960 \mathrm{~s}^{2,3}$, the technology is far from mature. Widespread commercial use has been limited, largely as a result of FCC restrictions. Due to concerns of interference with existing communication and navigation systems, the FCC limits UWB frequency bands and output power. Medical applications are limited to the 3.1 to $10.6 \mathrm{GHz}^{\mathrm{range}}{ }^{1}$. Throughwall imaging applications may operate below $960 \mathrm{MHz}$, or in the 1.99 to $10.6 \mathrm{GHz}$ range ${ }^{1}$. Commercial devices are currently limited in power, restricting the operating range to about ten meters ${ }^{4}$. Further studies are being performed to quantify the interference risks of UWB to conventional platforms ${ }^{5,6,7}$. Prototype systems have been developed at Lawrence Livermore National Laboratory using an experimental license.

\section{Narrowband System}

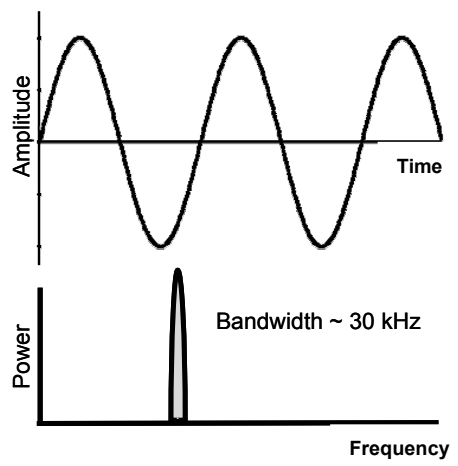

Ultra-wideband System

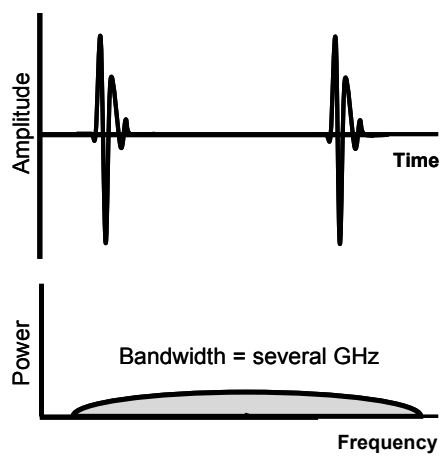

Figure 1: Narrowband vs. UWB in Time and Frequency Domain

\subsection{Ultra-wideband Advantages}

This section describes the unique advantages that make ultra-wideband systems beneficial for medical use.

\subsubsection{Penetrates biological tissues}

UWB signals are capable of penetrating a great variety of materials, including plastic, wood, rubber, sheetrock, dry soil, glass, and concrete ${ }^{8,9,10}$. In general, systems with lower center frequencies achieve better material penetration. Biological materials including skin, muscle, fat, and bone can also be penetrated, although not as easily as most low conductivity building materials. Highly conductive materials, such as metals and seawater, cannot be penetrated ${ }^{4}$.

\subsubsection{Non-invasive, non-contact, and non-ionizing}

UWB imaging systems would allow a physician to monitor internal organ movements without invasive surgical procedures. Unlike traditional ultrasound systems, which require direct skin contact, UWB sensors and imaging systems can operate at a standoff distance. Since UWB signals are non-ionizing, they do not cause the adverse effects associated with X-ray systems such as CT scanners. UWB imaging may enable physicians to make a preliminary diagnosis without subjecting the patient to risk or discomfort. In addition, these sensors are well suited for continuous patient monitoring to identify baseline changes.

\subsubsection{Low power, portable, and low cost}

While a CT scanner provides excellent image specificity, it is large and can cost several hundred thousand to over a million dollars. Handheld UWB sensor prototypes developed at Lawrence Livermore National Laboratory run on a single 9-V battery, and cost only hundreds of dollars to manufacture. Due to their portability and low cost, UWB sensors are ideal candidates for use in rural or remote settings and in-field use by first responders.

\section{SENSING WITH ULTRA-WIDEBAND}

A family of ultra-wideband sensors known as micro-power impulse $\operatorname{radar}^{11,12}$ (MIR) was developed at Lawrence Livermore National Laboratory. MIR sensors emit extremely narrow electromagnetic pulses and analyze received reflection signals for characteristic indicators of material boundaries and movements. MIR sensors are safe for medical 
use, since UWB signals are non-ionizing and emitted power is very low. In a typical MIR sensor used for medical applications, peak transmit power is 60 milliwatts and average transmit power is 25 microwatts.

The basic components of an MIR radar system include a transmitter, a reflective target, a receiver, and a signal processor [Figure 2]. The transmitter generates a series of short pulses. The shape of transmitted pulses is determined largely by the characteristics of the antenna. At dielectric interfaces, portions of the transmitted pulse reflect back toward the antenna. The receiver uses a range gate to sample the echo signals during a specific time interval corresponding to the round trip time of flight from the transmitter to the target, and back to the receiver'. A sensor with a fixed range gate can only detect echo signals from a single radial distance. By sweeping the range gate across a time span, or equivalent time sampling ${ }^{9}$, targets can be detected within a specified distance range. Multiple pulses are integrated to achieve a sufficient signal-to-noise ratio. Signal processing of the received pulse echoes may be performed in analog circuitry, using an FPGA, or using software algorithms running on a computer.

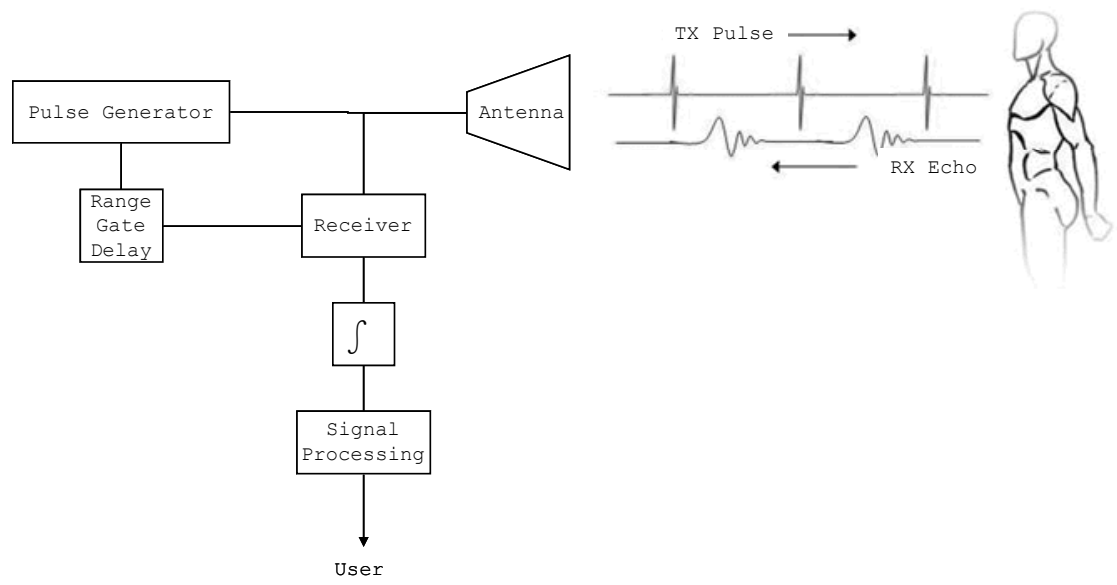

Figure 2: Micro-power Impulse Radar Sensor Block Diagram

\subsection{Distance Measurement}

One application of UWB radar is to measure the distance between the radar source and a reflective target. Ranging applications are based on measuring the round trip time of flight of a transmitted pulse. For example, if the time between sending a pulse and receiving its backscatter echo is $20 \mathrm{~ns}$, we can infer that the signal was reflected at the target after $10 \mathrm{~ns}$. If the target is in air, we can assume that the pulse velocity, $v$, is $3.0 \times 10^{8} \mathrm{~m} / \mathrm{s}$, the speed of light. Using the simple relationship, $d=v t$, we find the reflecting target was positioned 3 meters away from the radar. By sweeping the range gate across a time span of $15 \mathrm{~ns}$ to $25 \mathrm{~ns}$, we can detect reflected pulses from targets within a distance range of 4.5 to 7.5 meters.

Distance measurements become challenging when the nature of target mediums is unknown. Since propagation velocity is material dependent, assumptions are made about the electromagnetic properties and thickness of target materials. For example, at $2 \mathrm{GHz}$, the relative permittivity, $\varepsilon_{\mathrm{r}}$, of muscle and fat are 5.5 and 4.5 , respectively ${ }^{13}$. The corresponding propagation velocities are $v_{\text {muscle }}=1.3 \times 10^{8} \mathrm{~m} / \mathrm{s}$ and $v_{\text {fat }}=1.4 \times 10^{8} \mathrm{~m} / \mathrm{s}$, and are found by:

$$
v=\frac{c}{\sqrt{\varepsilon_{r}}}
$$

In several UWB medical applications, ranging sensors are used to identify physical abnormalities or deviations from a baseline, giving only approximate location information. For these applications, knowledge of exact thicknesses and material properties is not required. For example, a quick identification of the presence or absence of internal trauma injuries, such as intracranial hematoma or pneumothorax, can assist medical personnel in making triage and treatment decisions when more sophisticated diagnosis methods are unavailable. 


\subsection{Motion Detection}

To detect motion, one can simply monitor a ranging sensor over time for changes in target position. A derivative circuit and a comparator can be used as a motion indicator. Using swept range radar, it is possible to determine the locations and deflection magnitudes of multiple moving targets. We have demonstrated several medical applications for UWB motion sensing, including monitoring of speech, respiratory, and cardiac motions $\mathrm{s}^{4,9,14}$. UWB motion detectors have also been used in search and rescue applications, to identify victims trapped in collapsed structures.

\subsection{Imaging with Ultra-wideband Sensor Arrays}

A single UWB ranging sensor can provide position and motion information in one dimension only. While it is possible to detect the radial distance between the radar and a target, a single sensor cannot determine the target angle. If two sensors are used, it is possible to determine the position of a target in two dimensions [Figure 2]. To obtain threedimensional positioning information, an array of three or more sensors is used. UWB sensor arrays have been used to image a variety of targets, including bridge decks, buried land mines, walls, and the human body.

\subsection{Ultra-wideband Radar for Biological Sensing}

As UWB signals travel through tissues, their amplitudes attenuate exponentially with the factor $e^{-\alpha z}$, where $\alpha$ is the attenuation constant of the tissue, and $z$ is distance. When signals reach an interface between two mediums with differing dielectric properties, a portion of the signal is transmitted through the boundary, and a portion is reflected. The transmitted and reflected signals are given by:

$$
E_{t}=\tau \cdot E_{i} \quad \text { and } \quad E_{r}=\Gamma \cdot E_{i},
$$

where $E_{i}$ is the incident wave, $\tau$ is the transmission coefficient, and $\Gamma$ is the reflection coefficient. The transmission and reflection coefficients are given by:

$$
\tau=\frac{2 \cdot \sqrt{\varepsilon_{r 1}}}{\sqrt{\varepsilon_{r 1}}+\sqrt{\varepsilon_{r 2}}} \quad \text { and } \quad \Gamma=\frac{\sqrt{\varepsilon_{r 1}}-\sqrt{\varepsilon_{r 2}}}{\sqrt{\varepsilon_{r 1}}+\sqrt{\varepsilon_{r 2}}}
$$

where $\varepsilon_{r 1}$ and $\varepsilon_{r 2}$ are the relative permittivities of the two mediums. ${ }^{14,16}$ By detecting ultra-wideband reflections, sensors can determine the presence or absence of abnormalities at tissue boundaries and monitor their motion.

For sensing biological tissue boundaries, adjacent tissues with differing dielectric properties are easiest to see. To illustrate this point, consider the task of identifying a pneumothorax, or collapsed lung. The relative permittivities of tissues in the chest cavity over typical UWB frequencies are shown [Figure 4]. There is a significant difference between the dielectric properties of an inflated lung compared to a deflated lung. Using the dielectric constants of muscle, inflated lung, and deflated lung at $2 \mathrm{GHz}$, we find the reflection coefficient at the muscle/inflated lung interface to be $\Gamma_{M / L L}=0.25$, and the reflection coefficient at the muscle/deflated lung interface to be $\Gamma_{M / D L}=0.03$. We can detect the presence of an abnormal condition, such as pneumothorax, by comparing echo signals from a healthy lung to an afflicted lung. The same principles are applied to other UWB medical applications, including cardiac and respiratory monitors, and intracranial hematoma detection. 


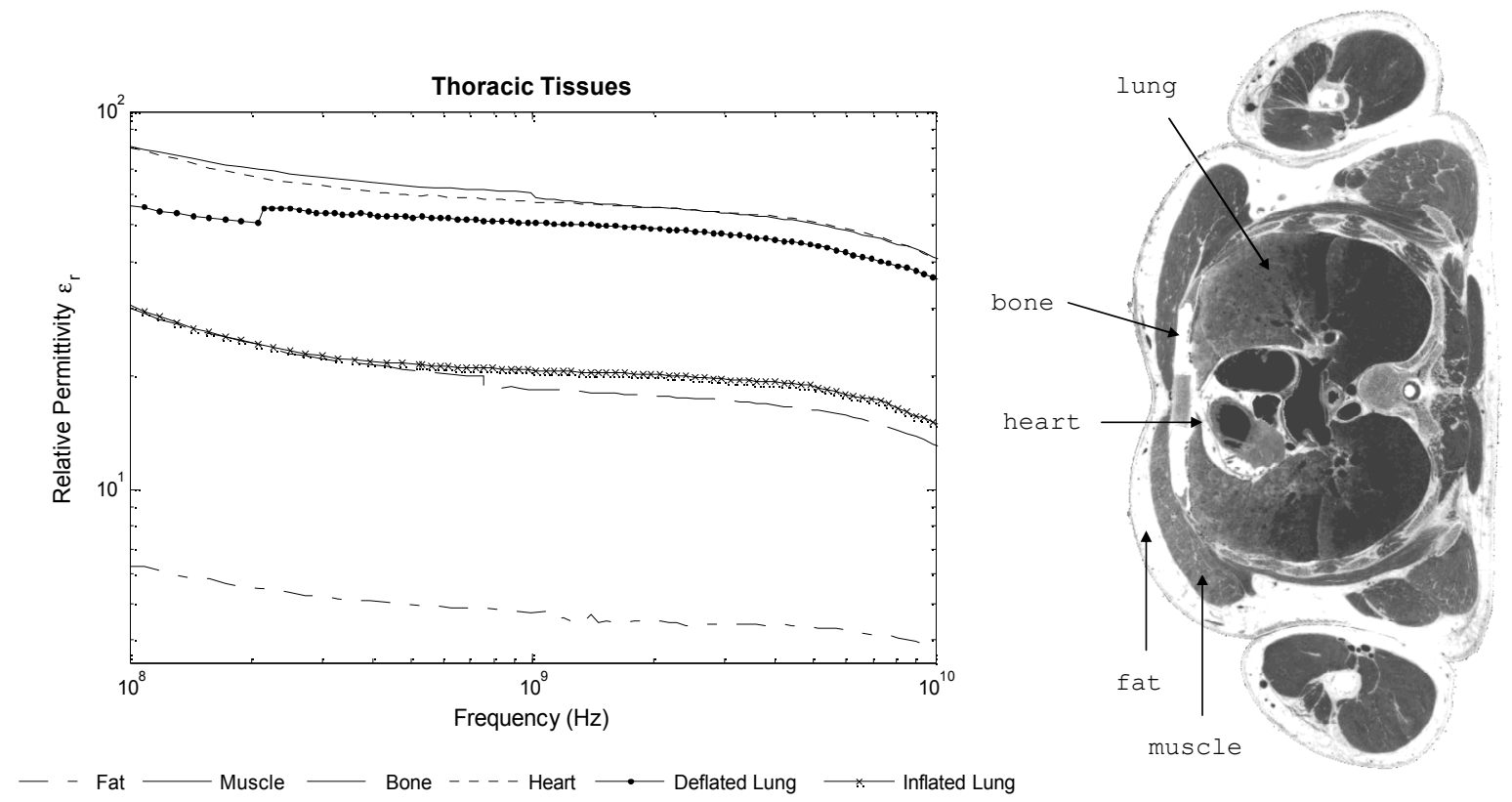

Figure 4: Thoracic Tissues ${ }^{17}$ and Dielectric Properties ${ }^{13}$

\subsection{Design Variables and Tradeoffs}

A designer of an UWB system must make a number of design choices specific to the application. These choices are interrelated, and reveal many tradeoffs in system performance. The following section outlines the key specifications involved in the design of an ultra-wideband sensing or imaging system, including frequency content, power, pulse repetition rate, receiver window size, and receiver integration time.

\subsubsection{Frequency Content of UWB Signals}

The frequency content of an ultra-wideband signal is specified by its center frequency and its bandwidth. A large bandwidth is beneficial, because many materials can be penetrated while achieving good spatial resolution. Choosing the frequency content of the system has implications for other system parameters. For example, low frequencies are desirable for achieving good penetration through dense materials. To create a lower frequency signals, however, a larger antenna is required, increasing the overall size of the device. High frequency signals yield good specificity, since the measurable spatial resolution is related proportionally to the wavelength. A very large bandwidth, however, can reduce the signal to noise ratio (SNR). For Gaussian noise, the noise power is proportional to signal bandwidth. Therefore, for a fixed signal power, as bandwidth increases, the SNR decreases.

\subsubsection{Power}

The power characteristics of an UWB system can be described by its instantaneous peak signal power, average signal power, and average system power consumption. A large peak signal power is important for obtaining good penetration through materials and long operating distances. An increase in peak power results in a proportional increase in the SNR. A major drawback, however, is a reduction in the efficiency of high power pulse generation circuits. As average system power consumption increases, the battery life decreases. For very high power systems, there could be concerns for interference with other radiofrequency devices and safety.

\subsubsection{Pulse Repetition Rate}

The repetition rate of emitted pulses sets an upper limit on the operating distance of UWB sensors. UWB systems measure the time of flight for pulses to reflect off of a target. The pulse repetition rate must be slow enough to allow reflected pulses to return to the receiver; otherwise, transmitted pulses would interfere with received signals. A faster pulse repetition rate improves the responsiveness of motion detection systems, allowing them to recognize high frequency movements such as vocal cord vibrations. To reduce the likelihood of being detected by or causing 
interference to narrowband systems and other ultra-wideband systems, randomized noise is added to the pulse repetition interval. This technique also reduces interference from other sources. ${ }^{9}$

\subsubsection{Receiver Window Size}

A range gate is used to sample received signals at specific time intervals. The receiver window size corresponds to the time window for sampling. Choosing a small window allows greater spatial resolution and better sensitivity to small movements, however, faster sampling circuits are required.

\subsubsection{Receiver Integration Time}

In order to improve the signal-to-noise ratio of an UWB system, averaging can be used. Instead of examining the received signal from a single pulse, which will be obscured by a number of noise sources, many pulses can be observed, one after another. These received signals are accumulated for some period of time, performing the equivalent of integration or averaging. The average received signal still contains noise, but its effects are greatly reduced. For purely Gaussian noise sources, the effective noise seen by the sensor is reduced by the square root of the number of cycles over which the integration is performed. This increases the operating range and sensitivity, but decreases the speed and responsiveness to motion.

\section{MEDICAL APPLICATIONS OF UWB RADAR}

The following are examples of ongoing research at Lawrence Livermore National Laboratory and collaborating institutions in the area of ultra-wideband radar technology applied to medical purposes. They illustrate the diversity and adaptability of the technology. Three different measurement techniques are represented: a swept range radar snapshot, motion characterization, and imaging.

\subsection{Hematoma Detector}

Patients having unrecognized intracranial injuries may develop permanent brain damage, severe disability, or death. Early diagnosis and treatment of intracranial hematomas is essential for improving patient outcomes ${ }^{17,19}$. The current method used to identify intracranial hemorrhage is computed tomography $(\mathrm{CT})^{20}$. Unfortunately, not all head trauma patients have access to this technology. Scanners are large, and therefore restricted to a hospital setting. Many rural hospitals cannot afford CT scanning equipment and support staff. CT scanners are not readily transportable; therefore patients must be transported to a hospital before a diagnosis can be made. This delay in neurosurgical intervention can result in irreversible brain injury or death.

A portable, hand-held hematoma detector could be of use for traumatic brain injury patients in the field to properly triage victims and minimize unnecessary delays before definitive medical intervention. Pre-hospital diagnosis may allow for earlier initiation of neuroprotective brain injury interventions, including operative hematoma evacuation, intracranial pressure monitoring and catheter placement, and drug therapy. Furthermore, such a portable device could screen for neurosurgical mass lesions in the multiple trauma patient too unstable for CT, or for hematomas in the intensive care unit setting. Expedited screening may facilitate the prevention of secondary brain injury consequent to hematoma development.

\subsubsection{Prototype Description}

A handheld micro-power impulse radar (MIR) prototype device was developed for detection of intracranial hematomas ${ }^{21}$ [Figure 5]. This system is based on an ultra-wideband swept range sensor. A swept range radar scans an area in space by varying the time delay between signal launch and capture. Using the propagation velocity, the time of flight can be related to distance. Key specifications are a center frequency of $2 \mathrm{GHz}$, a 200 ps pulse width, a 2 $\mathrm{MHz}$ pulse repetition rate, an average power density of less than 0.001 $\mathrm{mW} / \mathrm{cm}^{2}$, and a peak power density of $2.5 \mathrm{~mW} / \mathrm{cm}^{2}$, measured at a distance of $1 \mathrm{~cm}$.

The concept of operation is to use the inherent bilateral symmetry of the

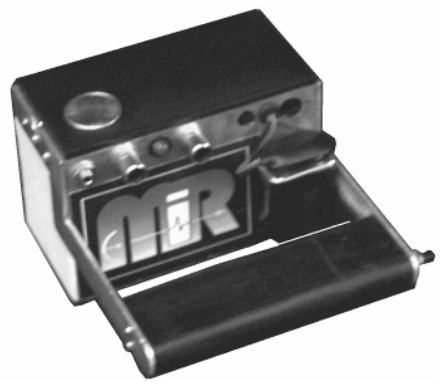

Figure 5: Handheld Hematoma Detector 
brain to identify abnormalities. In healthy patients, UWB echo signals from the left and right side of the brain should look very similar. If an injury is present, the signals will differ.

\subsubsection{Experiments}

The feasibility of hematoma detection with ultra-wideband radar has been tested using laboratory models and limited human subject experiments. The goal of early experiments was to determine whether a correlation exists between the magnitude of a simulated hematoma and UWB echo signals recorded by the MIR sensor. In the laboratory model, a phantom hematoma setup consisted of four pig brains placed inside of a human skull. Known volumes of either blood or air were injected between the skull and the brain tissue. In the human subject experiment, MIR sensor scans were collected from six healthy volunteers and six patients with intracranial injuries. The intracranial abnormalities included chronic subdural hematoma, acute subdural hematoma, acute epidural hematoma, and intracranial air (after hematoma drainage). Signals were analyzed by an individual who was blinded to the conditions of the subjects.

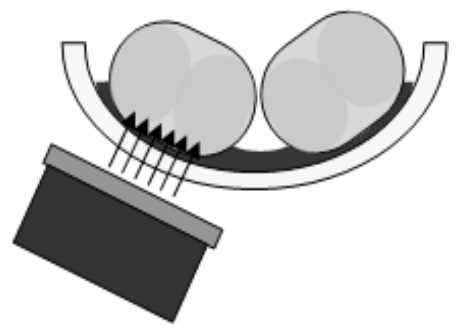

Figure 6: Hematoma Phantom

\subsubsection{Results}

In laboratory model experiments, characteristic features were observed in UWB echo signals corresponding to reflections from tissue interfaces. As blood was introduced between the skull and brain tissue, signal attenuation and delays were observed in certain characteristic features of the signal. An increasing spatial offset between brain tissue and the skull correlates with echo signal variations. MIR sensor signal returns from the phantom hematoma model with varying volumes of blood are shown [Figure 7]. As expected, reflections from the skull are well aligned for each echo trace. As the blood volume increases, a greater delay is introduced by the slower propagation of the signal through blood. The magnitude of the reflected signal is also decreased by the presence of hematoma.

In human subject experiments, a characteristic UWB echo signal was observed. Bilateral symmetry was confirmed in healthy patients. Delays and attenuations in characteristic features of the signal were observed in patients with hematoma [Figure 8]. A blinded observer correctly classified MIR scans as healthy or containing hematoma, and correctly predicted the location of intracranial abnormalities.

Based on the promising results of the laboratory and human subject experiments, an expanded collaborative study is proposed involving several hospitals and many patients. The goal is to determine whether the MIR sensor can effectively screen blunt head injury patients for urgent neurosurgical intervention.

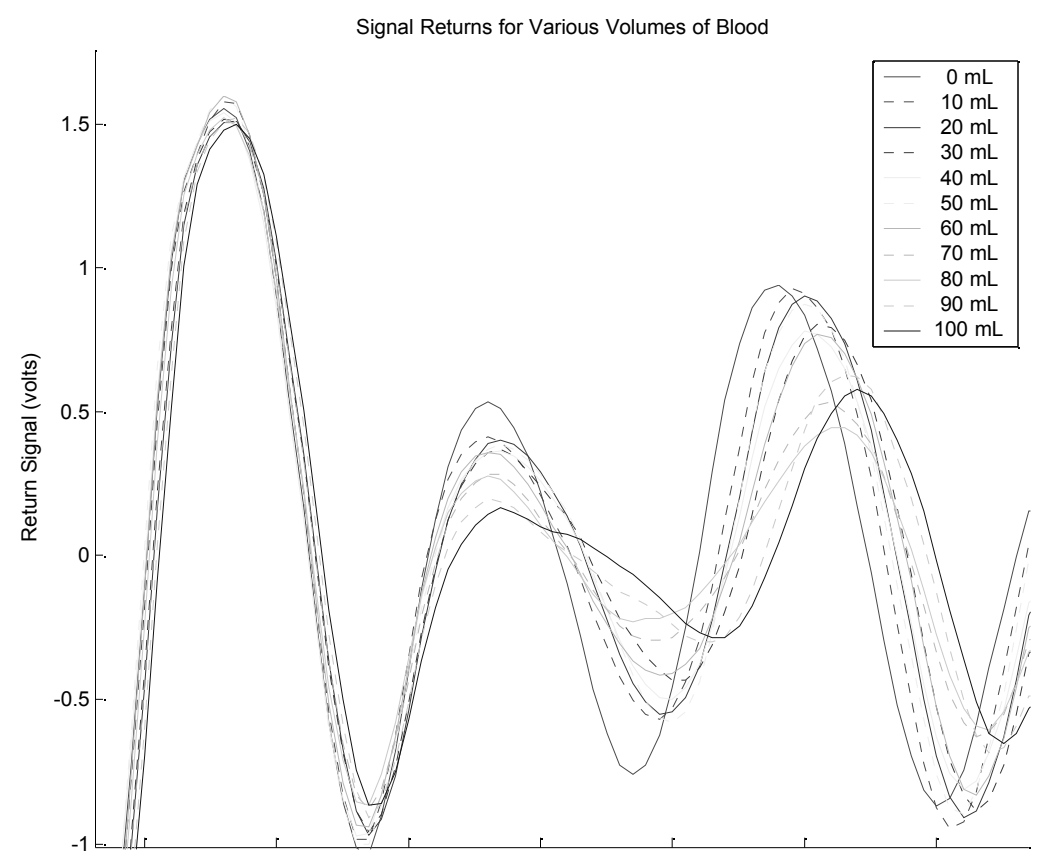

Figure 7: MIR signals from phantom hematoma 
(A)

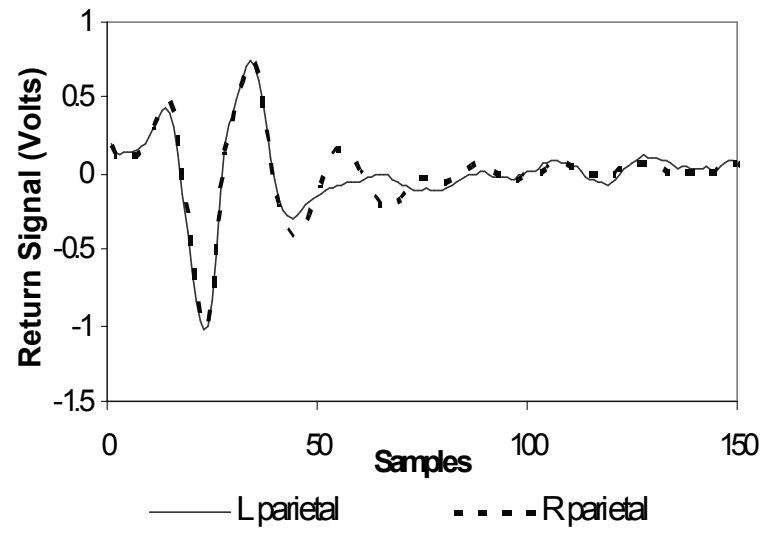

(B)

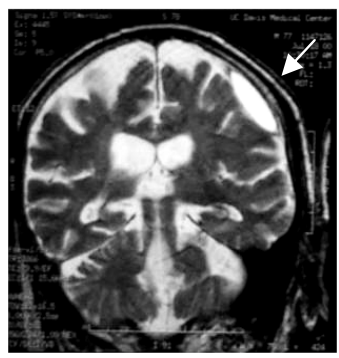

(C)

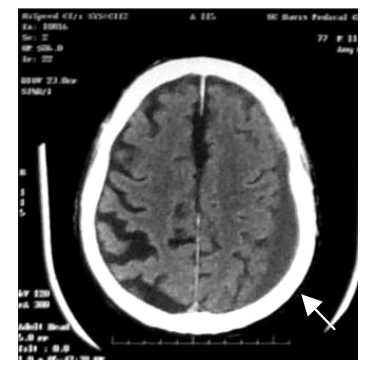

Figure 8: Patient with Chronic Subdural Hematoma - (A) MIR scan

(B) CT axial

(C) MRI coronal

\subsection{UWB Vital Signs Monitors}

Continuous, un-tethered monitoring of patient vital signs is not available. The primary tool used in screening for cardiovascular disease is the electrocardiogram (ECG), which records the electrical signals of the heart. It typically requires placement of twelve electrodes on the body. Heart rate monitors are smaller versions of an ECG, which use two or three electrodes to determine the heart rate. Another tool, which is frequently used in hospital settings to detect hypoxia, is the pulse oximeter. As a patient's hemoglobin saturation drops, blood becomes bluer in color. A pulse oximeter measures the amount of oxygen in a patient's blood by observing light reflections. As blood vessels expand and contract with every heart beat, the pulse oximeter signal fluctuates. The pulse oximeter probe requires direct contact with the finger or ear lobe. The stethoscope is an acoustic tool that enables physicians to listen to heart sounds and breathing. All of these tools require direct patient contact.

UWB sensors could provide a continuous, non-contact technique for cardiac and respiratory assessment that would be beneficial for minimizing patient disruption. Hourly collection of vital signs is perceived to be a leading cause of sleep deprivation in intensive care unit patients ${ }^{21}$. Studies have shown that sleep deprivation can slow the healing process by impairing protein synthesis, cell division, and cellular immunity ${ }^{23}$. Another application for non-contact vital signs monitoring may be in patients with chronic kidney disease. Sudden cardiac death accounts for about half of outpatient dialysis deaths ${ }^{24}$. Nursing staff must closely monitor patient vital signs for any irregularities. A continuous, non-contact cardiac and respiratory monitoring device may improve patient care without causing discomfort to patients. Because no human contact is required, the device would not require cleanings or sterilization. An ultra-wideband motion detection device could accurately monitor cardiac and respiratory movements, and could simply be mounted on the back of a chair or bed.

Due to its portability and low cost, UWB technology may be beneficial for use by first responders and medical personnel in remote areas. In field operations, a portable non-contact vital signs monitor could be useful for preliminary diagnostics and triage. Battlefield medics could use UWB cardiac monitors when an ECG was not available. Sensors could be worn by soldiers or firefighters in dangerous situations and monitored remotely. A sensor that can penetrate common building materials to detect respiration and body movement would be useful in search and rescue operations to identify live victims in burning buildings or collapsed structures. Other proposed applications include monitoring for Sudden Infant Death Syndrome and sleep apnea.

\subsubsection{Prototype Description}

Cardiac and respiratory monitoring has been achieved using the same hardware as described for hematoma detection [Section 3.1.1]. The primary differences are in signal processing. Instead of taking a snapshot, pulse echo signals are monitored continuously for signal changes in time. By differencing subsequent scans, motion can be identified. Filters 
can be used to target specific respiratory or cardiac frequencies. Swept range radars are used to generate intensity or waterfall plots as a function of time and depth. Fixed range sensors monitor changes in signal intensities a fixed distance from the radar.

\subsubsection{Experiments}

Vital signs were collected from 40 human volunteers at Walter Reed Army Institute of Research using a micro-power impulse radar range finding prototype. Readings from an ECG and pulse oximeter were captured simultaneously with MIR readings. These traditional sensor technologies were used to confirm MIR signal features were well correlated with respiration and cardiac events. MIR sensor readings were collected from each volunteer in four different body positions: standing upright, lying face up, lying on the right side, and lying face down. Since the readings of the MIR range finder prototype correspond to reflections off of tissue interfaces, rather than electrical impulses as in the ECG, body position was expected to be an important factor. Volunteers were asked to breathe slowly, while readings were captured for 60 seconds.

In another experiment, fixed range radar signals were compared to ECG, cardiac impedance, and acoustic heart signals to determine how well characteristic features of MIR signals could be related to traditional sensor technologies. Further experimentation needs to be performed in order to correlate radar signals to heart motions, such as cardiac contractions and arterial wall movements. Expanded human subject studies could reveal whether the MIR prototype has a high enough degree of sensitivity and specificity to detect the presence of cardiac arrhythmia or damage to the heart muscle.

\subsubsection{Results}

Using the range finding MIR prototype, the human respiration and cardiac cycle can be clearly identified. A depth scan is shown as an intensity plot, where the $\mathrm{Y}$-axis can be interpreted as depth (distance from the radar) and the $\mathrm{X}$-axis is time [Figure 9]. Intensity corresponds to the magnitude of the received signal. Low frequency undulations correspond to slow respiration, while high frequency features correspond to heart motion. ECG and pulse oximeter traces are well correlated with the periodic features seen in the MIR depth scan.
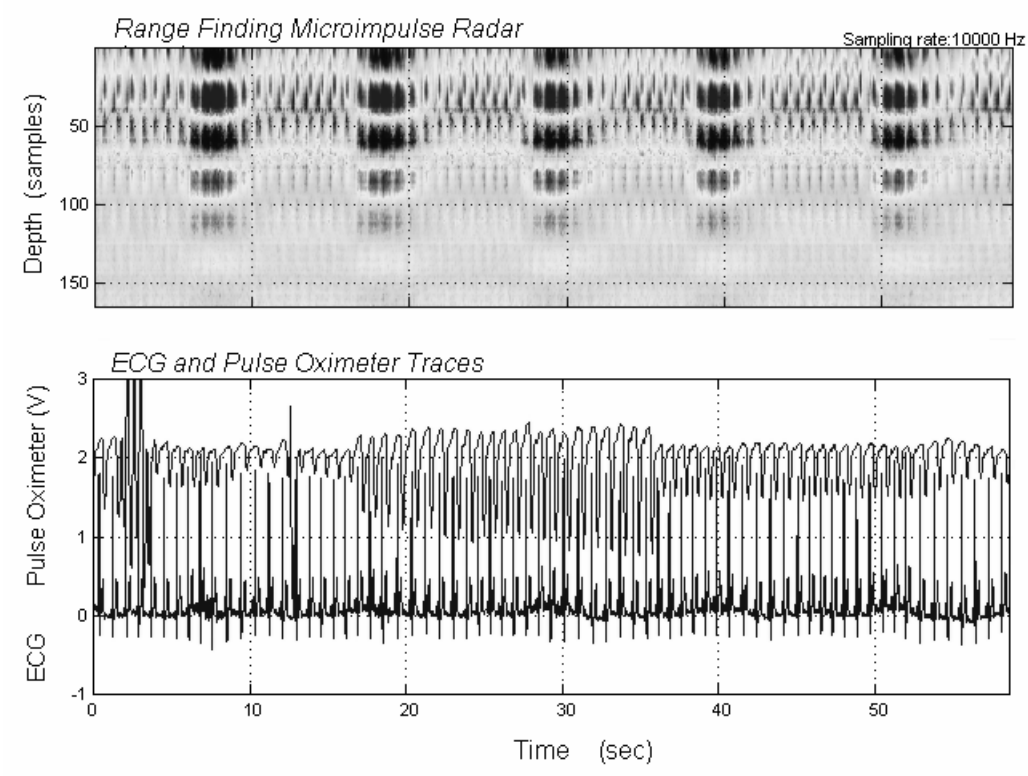

Figure 9: UWB Vital Signs Monitor, ECG and Pulse Oximeter Signals from Human Volunteer

Return signals from the fixed range sensor also correlate well with traditional cardiac monitoring methods. A comparison of traces from the ultra-wideband radar, impedance sensor, acoustic sensor, and ECG are shown [Figure 10]. We have demonstrated the feasibility of using ultra-wideband technologies to monitor cardiac and respiratory 
motions; however more investigation is required to understand the relationship between UWB radar signals and advanced cardiac functions. UWB Radar provides information about a fundamentally different phenomenon than an ECG; it is related to the actual motions of the heart muscle rather than its electrical impulses. Therefore, it is conceivable that UWB radar technology may reveal diagnostic capabilities not readily available in traditional methods.

\subsection{Ultra-wideband imaging of the human body}

Traditional techniques for imaging the human body include ultrasound, magnetic resonance imaging (MRI), computed tomography (CT), and traditional x-rays. UWB imaging may provide a means of long term continuous monitoring of the human body that cannot be accomplished using traditional techniques. CT scanners and $\mathrm{x}$-rays emit ionizing radiation, and exposure should be minimized. Ultrasound requires direct skin contact, and is therefore suited for short term monitoring only. Magnetic resonance imaging is not practical for long term continuous monitoring because the mechanics of

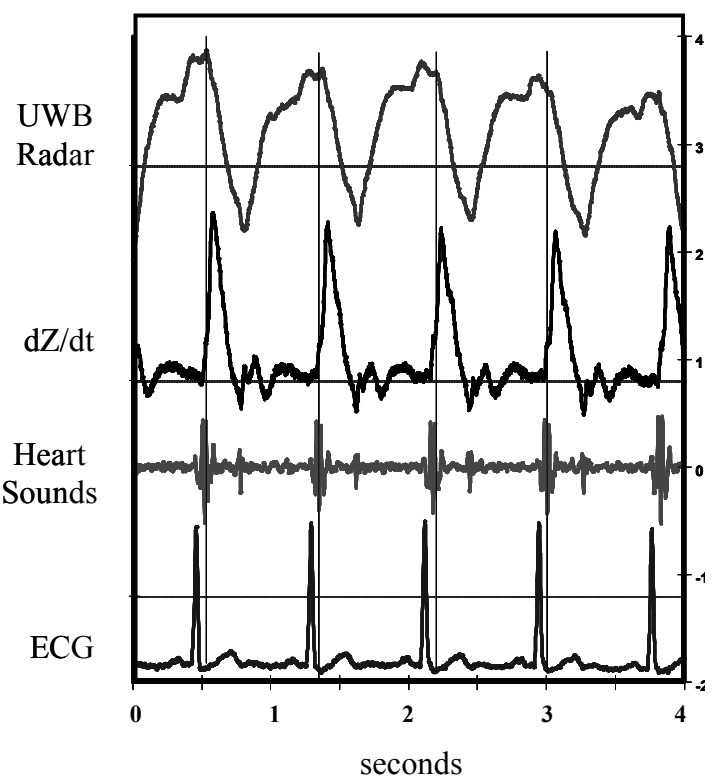

Figure 10: Comparison of UWB Cardiac Signals to Traditional Methods

the instrument surround the patient. Also, metallic objects are prohibited, which excludes individuals with a surgical prosthesis or pace maker.

An UWB imaging instrument does not emit ionizing radiation, does not require patient contact, and can be operated at a standoff distance, and therefore may be suitable for long term, continuous monitoring purposes. Such an instrument could be useful for monitoring a patient in a hospital bed, as an alternative to CT or MRI when a patient is not stable enough to be relocated, or for real-time imaging of organs. Also, embedded metallic objects are easily identified.

\subsubsection{Prototype Description}

The imaging instrument consists of a phased array of swept range UWB radar sensors. A digital timing and data acquisition system is used to control the launch and capture times of each sensor. The system is electronically reconfigurable, such that the size and resolution of the scanned volume can be adjusted. The achievable resolution is related to the frequency content of the radar elements and the array aperture. The signal to noise ratio is given by both the number of elements and their spatial distribution, as well as the standoff distance. Simulations suggest that a resolution of $1 \mathrm{~cm}$ can be obtained for targets positioned at a 1-meter standoff with an array of 48 sensors spanning a 1meter by 1 -meter aperture. Raw data for a $200 \times 200$ pixel image is captured at 20 frames per second. Using tomographic reconstruction algorithms, a two-dimensional image is created from scans of a volume in space. Images are reconstructed in post-processing on a separate computer. A field programmable gate array (FPGA) implementation to carry out the image reconstruction is currently under development. We estimate the FPGA system will be capable of processing four reconstructed images per second at the output quality exhibited by Figure 11.

\subsubsection{Experiments}

A preliminary experiment used an UWB radar array to scan a 1-meter by 2-meter by 0.5 -meter volume. Human volunteers were imaged at a standoff distance of one meter. To simplify the experiment, the human subjects were scanned in front of radar absorbent foam.

\subsubsection{Results}

Constructed images show recognizable human features. A female and a male subject are shown [Figure 11]. The male was wearing a metallic belt buckle, which appears as a highly reflective object. The image of the female is $300 \times 220$ pixels. Each pixel corresponds to $0.6-\mathrm{cm}$. The image of the male is $485 \times 320$ pixels. Each pixel corresponds to 0.4$\mathrm{cm}$. 
Although these results do not demonstrate the sub-millimeter specificity of an MRI or CT scan, higher resolutions could be obtained through the use of a higher frequency radar or a greater number of radar elements. Ultra-wideband radar imaging has potential to be a viable patient monitoring technology. Key advantages that satisfy specific application needs not addressed by MRI or CT include real-time imaging of moving organs, inexpensive hardware, and portability.
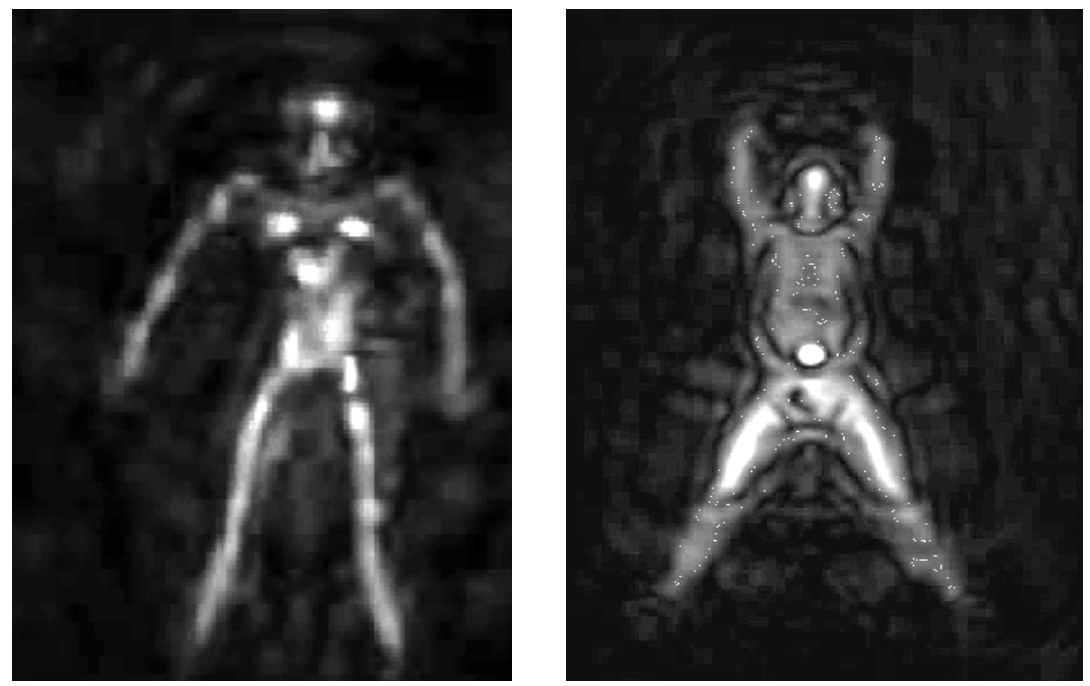

Figure 11: UWB radar images of human volunteers. Female (left) Male (right)

\section{CONCLUSION}

Ultra wideband radar is a flexible technology that can be adapted to a variety of medical applications. A suite of Micropower Impulse Radar technologies have been developed at Lawrence Livermore National Laboratory. Prototype devices have the ability to detect internal tissue boundaries, monitor motion, and produce images without direct contact with a patient. They are low-power, safe, portable, and inexpensive. Through the selection of design parameters, UWB radar systems can be tailored to achieve high performance for specific applications. We have demonstrated the feasibility of MIR devices for use in detection of internal injuries, such as hematoma. The hand-held form factor, low power requirement, and rapidly generated results make this detector suitable for use in remote areas, where CT and MRI imaging systems are unavailable. Another adaptation of an MIR sensor was used to monitor respiratory and cardiac motion in human subjects. Potential applications include use by first responders for diagnosis and triage and unobtrusive vital signs monitoring in hospitals and dialysis clinics. Multiple radar sensors can be combined in a phased array and used to scan a three-dimensional volume for imaging purposes. Preliminary experiments show successful tomographic reconstruction of UWB signals for imaging human subjects. Unlike CT and MRI systems, ultra-wideband radar could be used for continuous, real-time monitoring over extended time periods. These examples demonstrate only a few possibilities for this promising technology.

\section{ACKNOWLEDGEMENTS}

This work was performed under the auspices of the U. S. Department of Energy by the University of California, Lawrence Livermore National Laboratory under Contract No. W-7405-Eng-48. UCRL\# XXXXX

We would like to acknowledge the following individuals involved in UWB radar research at LLNL: Vickie Abreu, Steve Azevedo, Dave Chambers, Greg Dallum, Farid Dowla, Richard Leach, Peter Haugen, Claudia Hertzog, Faranak Nekoogar, Dennis Matthews, Garth Pratt, Alex Spiridon, Jim Trebes, Pat Welsh, and Jim Zumstein. UC Davis Medical Center collaborated in hematoma studies. Cardiac and respiratory monitoring experiments were performed at the Walter Reed Army Institute of Research, the Renal Research Institute, and the Beth Israel Medical Center. 


\section{REFERENCES}

1. Code of Federal Regulations: Part 15 Subpart F Ultra-Wideband Operation. Federal Communications Commission. May 2002.

2. T.Barret, "History of UltraWideBand (UWB) Radar \& Communications: Pioneers and Innovators", Progress in Electromagnetics Symposium, Cambridge, MA, July 2000

3. R.J.Fontana. "Recent System Applications of Short-Pulse Ultra-Wideband (UWB) Technology" IEEE Transactions on Microwave Theory and Techniques. Vol.52 No.9. Sept 2004

4. S.Azevedo, "Exploring the Ultrawideband" Science and Technology Review. UCRL-TR-52000-04-9. Sept 2004.

5. J.R.Hoffman, M.G.Cotton, R.J.Achatz, R.N.Statz, R.A.Dalke "Measurements to Determine Potential Interference to GPS Receivers from Ultrawideband Transmission Systems" NTIA Report 01-384. February 2001

6. J.E.Padgett, J.C.Koshy, A.A.Triolo, "Physical-Layer Modeling of UWB Interference Effects" DARPA NETEX Program MDA972-02-C-0056. Jan 2003.

7. J.R.Fontana, "An Insight Into UWB Interference From a Shot Noise Perspective" IEEE Conference on Ultra Wideband Systems and Technologies Baltimore, MD. May 2002

8. A.Safaai-Jazi, S.M.Riad, A.Muqaibel, A.Bayram. "Ultra-wideband Propagation Measurements and Channel Modeling: Report on Through-the-Wall Propagation and Material Characterization" DARPA NETEX Program. Nov 2002. Sept 2005. http://www.darpa.mil/ato/programs/netex/models.pdf

9. S.G.Azevedo and T.E. McEwan, "Micropower Impulse Radar" Science and Technology Review, UCRL-52000-961/2, Jan. 1996

10. R.Dilsavor, W.Ailes, P.Rush, F.Ahmad, W.Keichel, G.Titi, M.Amin. "Experiments on wideband through the wall imaging." Proceedings of the SPIE Defense \& Security Symposium, Conference on Algorithms for Synthetic Aperture Radar Imagery XII, Orlando, FL, March 2005

11. T.McEwan, "Ultra-Wideband Radar Motion Sensor". U.S. Patent 5,361,070, Nov. 1, 1994

12. T.McEwan, "Impulse Radar with Swept Range Gate". U.S. Patent 5,805,110, Sept. 81998

13. C.Gabriel, "Compilation of the Dielectric Properties of Body Tissues at RF and Microwave Frequencies" Brooks Air Force Technical Report AL/OE-TR-1996-0037. Appendix D. June 1996

14. J.Mast, S.Azevedo, W.Haddad, L.Ng, G.Burnett, "Micropower Impulse Radar Technology and Applications" Lawrence Livermore National Laboratory Technical Report UCRL-ID-130474. Apr. 1998

15. S.J.Orfanidis. Electromagnetic Waves and Antennas. Ch.2, Ch.4. June 2004 $<\mathrm{http}: / /$ www.ece.rutgers.edu/ orfanidi/ewa $>$

16. S.Ramo, J.R.Whinnery, T.Van Duzer. Fields and Waves in Communication Electronics. pp270-316.John Wiley \& Sons USA. 1984

17. Visible Human Project. US National Library of Medicine. National Institutes of Heath. Sept 2003. $<$ http://www.nlm.nih.gov/research/visible/>

18. P.E.Stieg, C.S.Kase. "Intracranial hemorrhage: diagnosis and emergency management." Neurology and Clinical Neurophysiology. pp273-290. May 1998

19. KY.Willman, DE.Bank, M.Senac, DL.Chadwick. "Restricting the Time of Injury in Fatal Inflicted Head Injuries". Child Abuse \& Neglect. pp929-940. Oct. 1997

20. CS.Zee, JL.Go, "CT of Head Trauma" Neuroimaging Clinics of North America. pp525-539. Aug 1998

21. W.Haddad, J.Chang, T.Rosenbury, G.Dallum, P.Welsh, D.Scott, D.Duarte, V.Acevedo-Bolton. "Microwave Hematoma Detector for the Rapid Assessment of Head Injuries" Lawrence Livermore National Laboratory Technical Report UCRL-ID-137901. Feb 2000

22. N.S.Freedman, N.Kotzer, R.J.Schwab. "Patient Perception of Sleep Quality and Etiology of Sleep Disruption in the Intensive Care Unit" American Journal of Respiratory and Critical Care Medicine. Vol 159. No.4. pp1155-1162. Apr. 1999

23. S.L.Krachman, G.E.D'Alonzo, G.J.Criner. "Sleep in the Intensive Care Unit" Chest. Vol 107. pp1713-1720. Oct 2005

24. C.A. Herzog, Q.Fan, A.J.Collins. "Epidemiology of Sudden Cardiac Death in Dialysis Patients in the United States, 1991-2001” American Society of Nephrology: USRDS Renal Week 2004. St. Louis, MO Oct 2004. 